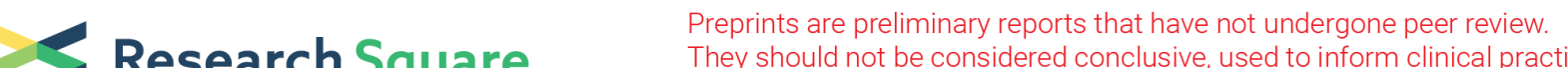 $\begin{array}{ll}\text { Research Square } & \text { They should not be considered conclusive, used to inform clinical practice, } \\ \text { or referenced by the media as validated information. }\end{array}$
}

\section{Trauma and Orthopedics in COVID-19 Pandemic: an Epidemiological Study in a Tertiary Center, South of Iran}

\section{Seyed Mohammad Tahami}

Shiraz University of Medical Sciences

Seyed Ali Hashemi

Shiraz University of Medical Sciences

Armin Akbarzadeh

Shiraz University of Medical Sciences

Mohammad Hadi Gerami

Shiraz University of Medical Sciences

\section{Amir Aminian}

Shiraz University of Medical Sciences

\section{Amirreza Mesbahi}

Shiraz University of Medical Sciences

\section{Amin Zarrabi}

Shiraz University of Medical Sciences

Rasool Safari ( $\square$ rasoolsafari1370@gmail.com )

Shiraz University of Medical Sciences https://orcid.org/0000-0002-3333-0896

\section{Research Article}

Keywords: Trauma, Orthopedics, COVID-19, Pandemic, Epidemiology

Posted Date: June 7th, 2021

DOI: https://doi.org/10.21203/rs.3.rs-576796/v1

License: (c) (1) This work is licensed under a Creative Commons Attribution 4.0 International License. Read Full License 


\section{Abstract}

Introduction: The novel COVID-19 disease was presented at first in Wuhan, China, in December 2019. During COVID-19 pandemic, approaching trauma patients, whom could have COVID-19, is a challenging issue required special consideration and healthcare setting.

Methods and materials: This retrospective cohort study was conducted at Emtiaz hospital, the main trauma center of the south of Iran, between March 2020 and August 2020 from the beginning of the COVID-19 pandemic. Trauma-related data such as the mechanism of trauma, injury pattern, and surgical intervention procedure of all the suspicious COVID-19 patients were documented. ICU admission and mortality rate were investigated as outcome variables.

Results: During six months of the pandemic period, 9248 patients were referred to our referral trauma center, with 222 patients suspicious of having COVID-19 infection. Among sixty-four cases, whom were confirmed to be positive, 33 orthopedic injured patients were observed. The mortality rate of COVID-19 positive cases was $15.6 \%$ (10 patients out of 64 ) and COVID-19 negative ones was $10.1 \%$ (16 patients out of 158) which was not statistically significant (p.value:0.25). Multivariate analysis of the effect of the baseline and trauma related factors on mortality rate, showed that older age ( $p$. value: 0.001), COVID-19 infection ( $p$. value: 0.033 ), and surgical procedure (p.value:0.038) are the poor prognostic factors associated with mortality rate.

Conclusion: The mortality rate of trauma patients with COVID-19 positive infection was $15.6 \%$ (10 patients). Older age, COVID-19 infection, and surgical procedure were observed as the statistically significant prognostic factors leading to more mortality rate.

\section{Level of Evidence: III}

\section{Introduction}

The novel coronavirus, called SARS-COV-2, cause of COVID-19 disease, was presented at first in Wuhan, China, in December 2019 [1]. Soon after that, it was spread out through more than 190 countries with devastating morbidities and mortalities with over 450,000 infected cases and 20,000 deaths, leading the World Health Organization (WHO) to announce this outbreak as pandemic and public health emergency by March $2020[2,3,4]$.

The governors and authorities of the affected countries tried their best to legislate every possible action to reduce caseloads, including lockdown measures, strict traffic laws, city blockade, social distancing, and self-isolation at homes to mitigate viral transmission through the vast number of people [2].

COVID-19 cases could be suspected by obtaining their medical history, exposure to the suspicious or infected cases in the past 14 days and observing clinical symptoms including fever, cough and dyspnea 
$[5,6]$. Confirmation of the diagnosis is recommended via performing PCR or lung CT scan, which have approximately $83.3 \%$ and $97.2 \%$ sensitivity, respectively [7].

During the COVID-19 pandemic, elective orthopedic surgeries were canceled or postponed and healthcare potential capacities and resources were reallocated and reorganized to support the large-scale number of infected patients with COVID-19. Urgent and emergent orthopedic situations, including trauma, infection, and malignant tumors were in priority besides the COVID-19 pandemic $[8,9,10]$.

Iran has a high number of motor vehicle accidents, accounting for approximately 20,590 events, annually. Despite lockdown measures and quarantine rule in the COVID-19 pandemic, a significant number of trauma patients referred to orthopedic centers [11]. Dealing with orthopedic trauma patients, whom could have COVID-19, is a challenging issue that requires special consideration and healthcare setting.

Our study aims to shed light on the characteristics of trauma and orthopedic patients with COVID-19 and eliciting different aspects of managing these cases in a single referral trauma center.

\section{Materials And Methods}

\section{Study design}

This retrospective cohort study was performed during six months from the beginning of the COVID-19 pandemic between March 2020 and August 2020. Data were collected from Emtiaz Hospital, the main trauma center of southern Iran, which is located at the Shiraz. Since the report of the novel COVID-19 infection in Iran on March 2020, all the trauma patients who are supposed to be admitted or to be observed for over six hours were considered as "probable" for COVID-19 infection.

Stable trauma patients were examined, and precise history was taken for any signs and symptoms of COVID-19 infection or recent exposure to a suspicious case of COVID-19. According to local protocols, all patients were screened by chest radiography, complete blood cell count, Erythrocyte sedimentation rate (ESR), and C-reactive protein (CRP), besides the mentioned history taking and physical examination. If there was any doubt or they had any findings in favor of COVID-19 infection, they were transferred to special gray zone ward dedicated to probable COVID-19 infected patients and PCR and lung Highresolution computed tomography (HRCT) scan were requested and screened for any possible signs of COVID-19 infection. All unstable patients were considered probable and transferred to a particular intensive care unit (ICU) and were evaluated for COVID-19 by further confirmatory tests such as PCR or lung HRCT.

\section{Data collection}

Data of the COVID-19 probable trauma patients were collected from their medical records. Baseline characteristics (including age, sex, comorbidities, history of opium or cigarette smoking) and COVID-19 related data (signs, symptoms and history of exposure to positive or suspicious individuals) were obtained from the registry that belongs to the center of control and prevention of COVID-19 in Fars 
province, southern of Iran. The trauma-related data including traumatic injuries, need for ICU admission, duration of hospitalization, andoutcome then synced with the mentioned information from their medical records.

\section{Laboratory and radiologic confirmation of COVID-19}

Nasopharyngeal and oropharyngeal samples were obtained and checked for SARS-CoV-2 using Real-time reverse transcriptase-polymerase chain reaction (RT-PCR). HRCT was obtained if the chest X-ray was suspicious. HRCTs were reviewed by a trained radiologist. The patients who had a diagnostic pattern of COVID-19 in HRCT or those with positive PCR test were considered as confirmed cases of COVID-19.

\section{Statistical analysis}

Data was analyzed by Statistical Package for the Social Sciences version 15.0 (SPSS Inc., Chicago, IL). Descriptive results are presented as mean \pm standard for quantitative data and number and percentile for qualitative data. The Chi-square test and T-test were applied to compare different parameters between probable trauma patients with positive and negative paraclinical test results. Univariate and multivariate analyses were used to determine the association of different risk factors, including age, mechanism of trauma, smoking and opium consumption, comorbidities, and COVID-19 infection with outcome factors such as ICU admission and mortality rate.

\section{Results}

During six months from the emerging COVID-19 pandemic, 9248 patients were visited in our referral trauma center. As shown in Figure-1, from 222 patients who were suspicious for COVID-19 infection, 64 trauma patients were confirmed by either a HRCT scan or PCR test. Thirty-three patients of the confirmed cases had an orthopedic injury that 23 needed surgical treatment.

Baseline characteristics of the trauma patients with suspicious COVID-19 infection, categorized into COVID-19 negative or positive groups based on their confirmatory tests, are shown in Table.1. The age and sex distribution were similar between the two groups. The most prevalent comorbidities were hypertension, cardiovascular disease, and diabetes mellitus in both groups. The baseline characteristics were similar between positive and negative cases.

Clinical and outcome characteristics of the two groups were presented in Table.2. Among all only history of exposure to a suspicious COVID-19 infectious case was significantly higher among the positive cases compared to the negative ones $(8(12.5 \%)$ versus $5(3.2 \%)$; p.value $=0.012))$. The most prevalent signs and symptoms among the patients in both groups were fever, cough, and weakness. One hundred eleven (48.1\%) patients had been admitted to ICU and 26 (11.3\%) were deceased.

Univariate analysis of the association of the baseline and prognostic factors, including, age, sex, mechanism of trauma, cigarette smoking, opium consumption, COVID-19 infection, and comorbidities with ICU admission was performed which revealed cigarette smoking is the only statistically significant 
factor associated with more ICU admission of the patients. After adjusting for the effect of all the mentioned variables, multivariate analysis showed no significant association of any of the parameters with ICU admission. (Table. 3)

Univariate analysis of the association of the baseline and prognostic factors, including age, sex, mechanism of trauma, cigarette smoking, opium consumption, COVID-19 infection, comorbidities and surgical procedure with mortality rate was performed which revealed older age and asthma as the only statistically significant factors associated with more mortality rate of the trauma patients. After adjusting for the effect of all the aforementioned variables, multivariate analysis showed a significant association of older age (p.value: 0.001$)$, COVID-19 positive (0.03), and surgical procedure (0.03) with more mortality rate. (Table. 4)

Table.5 illustrates the characteristics of the trauma patients who needed orthopedic intervention. Twentyfour $(72.7 \%)$ cases had traffic accidents while the remaining $9(27.3 \%)$ ones happened by falling. The most prevalent sites of injury were pelvic and acetabulum (10 cases), femur (11 cases), and clavicle (7 cases). Twenty-three patients were treated surgically with a mean admission to surgery duration of $8.2 \pm$ 6.8 days. The mean operation duration time was $3.1 \pm 2.0$ hours.

\section{Discussion}

\section{Characteristics of COVID-19 and trauma patients}

Among all of 9248 trauma patients, 222 cases were suspicious of having COVID-19 infection. Similar to previous reports, fever, cough and fatigue were the most common symptoms in our study $(39 \%, 13 \%$, $13 \%$, respectively) $[10,12,13,14]$.

Sixty-four confirmed cases showed a history of exposure to suspicious and symptomatic people at a higher rate compared to the 158 COVID-19-negative cases, which was statistically significant. $(12.5 \%$ to $3.2 \%$. p.Value $=0.012$ ). This could lead us to be more cautious about approaching a patient with a history of exposure by not only providing more personal protective equipment for medical staff, but also taking immediate actions toward the isolation of these patients from other unsuspicious ones.

The most prevalent comorbidities among our studied patients were hypertension, diabetes mellitus and cardiovascular disease, similar to the previous reports $[13,15,16]$.

We observed different age and injury patterns compared to the other studies in COVID-19 pandemic reporting the mean age of 67 years or older $[10,15,16]$, and demonstrating low energy trauma as the most common mechanism of trauma $[12,17,18]$. Mean age of the patients in our study was 42.5 and 80 $\%$ of them were men, most were the victims of motor vehicle accidents (MVA) (72.7\%). Besides, One study from Iran showed the mean age of the studied patients was 38.6, and high energy trauma was the main cause (8 patients out of 13) [11]. Noteworthy, it could be concluded that despite government legislation of 
lockdown measures, strict traffic laws, and self-isolation at home, the MVA still has a devastating impact and the utmost role, giving rise to traumatic injury to the patients in Iran during the COVID-19 pandemic.

Among 64 confirmed cases, 33 showed orthopedic injuries, mostly comprised of the pelvis and acetabulum, clavicle, and pertrochanteric fractures (10, 7, and 6 patients, respectively). Among the patients with orthopedic injuries, $24(72.7 \%)$ ones were the victim of MVA and 9 patients were injured due to falling down. The most common orthopedic injury in MVA and falling down subgroups were pelvic fractures and pertrochanteric fractures respectively. One systematic review on 44 COVID-19 positive cases with concomitant fractures, showed 29 patients with proximal femoral fractures, eight patients with spine fractures, and seven patients with limb bone fractures [16]. In our study, 23 (69\%) out of 33 COVID-19 positive cases with concomitant orthopedic injury were managed surgically in line with the result of the aforementioned review that 29 patients (68\%) out of 44 patients underwent surgeries [16].

\section{The outcome of COVID-19 and Trauma patients: ICU admission and hospital associated mortality}

111 patients (50\%) were admitted to the intensive care unit (ICU). This proportion remained constant among both COVID-19 negative and positive groups. One study of 82 COVID-19 positive versus 340 COVID-19 negative patients with concomitant hip fracture showed $9.8 \%$ (8 patients) ICU admission in COVID-19 positive case compared to $0.9 \%$ (3 patients) in COVID-19 negative ones, not presenting poor prognostic factors associated with more ICU admission [10]. We observed no significant association with any baseline and prognostic factors with more ICU admission. While, a study of 34 COVID-19 patients, who had been operated, with an ICU admission proportion similar to our study (44\%), stated that patients with comorbidities such as HTN and CVD, older age and the more complicated surgery have increased risk for admitting to the ICU [13]. Of note, the ICU admission rate among COVID-19 patients without surgery is documented at $26 \%$ in the literature [19]. While the proposed reason for the mentioned results of previous reports could be that any surgical procedure brings about immune system compromise and jeopardize multi-organ functions resulting in the need for more ICU care, it should be taken into account that our surgical procedures were performed after ICU admission of the trauma patients. One explanation for the observed nonsignificant effect of associated comorbidities on the rate of ICU admission among our patients, may be rooted in the lower mean of their age compared with the aforementioned studies.

\section{$11.7 \%$ (26 patients) of 222 patients were expired during hospitalization. The hospital-associated} mortality rate of the COVID-19 positive cases with concomitant orthopedic trauma was $15.6 \%$ (10 patients) which was in contrast to previous reports by Jain (36.3\%), Kayani (30.5\%), and Maniscalco (43

$\%)[10,16,20]$. The most logical explanation could be the younger population of our study (mean age: 42 ) comparing to the mentioned studies. One study on 34 COVID-19 patients who underwent surgery with closer age distribution (median age: 55 ) to ours, showed a $20.5 \%$ mortality rate [13]. Meanwhile, it should be mentioned that case fatality rate of COVID-19 patients without surgery is $2.3 \%$ [21]. After a multivariate analysis of risk factors association with the mortality rate in the suspicious COVID-19 patients with trauma, it was determined that older age, COVID-19 infection, and surgical procedure are 
poor prognostic factors. However, Kayani et al. showed cigarette smoking and multiple comorbidities, and Lei et al. proposed more complicated surgeries and medical comorbidities as detrimental factors associated with more mortality rate in their study population $[10,13]$. One of the most important findings of our study was that the presence of COVID-19 showed significant association with more mortality rate among trauma patients, which was similar to Kayani's report [10].

\section{Strengths and limitations}

We conducted a retrospective cohort study with a medium-sized sample of suspicious COVID-19 and trauma patients in a center with a high volume of incoming trauma patients from south of Iran, which evaluated 9248 trauma cases in a six months pandemic period. This large-scale referred patients to our hospital is an efficient representative of the Iranian people's profile regarding COVID-19 status during the pandemic. The other strength of our study could be using two confirming tests (HRCT and PCR) to increase more diagnostic accuracy and sensitivity.

One of the limitations was that asymptomatic carriers of COVID-19 disease were not investigated due to the lack of enough available PCR kits. Meanwhile, the incubation period of COVID-19 infection is stated to be 14 days, and trauma patients need to be managed urgently and any delay in surgical treatment may accelerate or exacerbate their current condition and leading to the poor functional outcomes [14, 22]. The other limitation was that any developing signs and symptoms in suspicious cases after their hospital course were not assessed.

During the COVID-19 pandemic, medical health care and hospital resources should be utilized wisely and optimally to reduce devastating morbidities and mortalities. The vast majority of trauma patients in Iran are victims of motor vehicle accidents and are in need of urgent medical and surgical interventions. Providing resourceful information about the epidemiological and clinical characteristics of trauma patients with COVID-19 would contribute to the well-organized decisions and systematic approach to the issues in pandemic condition.

\section{Conclusion}

Among 222 trauma patients with suspicious COVID-19 infection, fever, coughand fatigue were the most presenting signs and symptoms. The most prevalent comorbidities were HTN, CVD, and DM. Of 64 patients with confirmed COVID-19, 33 ones presented with orthopedic injuries, with the pelvic and acetabulum fractures as the most common injury. $48 \%$ of the COVID-19 positive patients were admitted to the ICU. The mortality rate of COVID-19 positive cases was $15.6 \%$ (10 patients). Older age, COVID-19 infection and surgical procedure were observed as the statistically significant prognostic factors leading to more mortality rate.

\section{Abbreviations}


WHO: World health organization; PCR: Polymerase chain reaction; CT: Computed tomography; HRCT:

High resolution computed tomography; ICU: intensive care unit; HTN: Hypertension; CVD:Cardiovascular disease; DM: Diabetes mellitus; SD: Standard deviation; Fx: Fracture,; ACL: Anterior cruciate ligament; Dx: Dislocation; MVA: Motor vehicle accidents

\section{Declarations}

\section{Funding}

The study has not any source of funding.

\section{Acknowledgements}

Bone and joint research center of orthopedic department, Shiraz university of medical sciences.

\section{Authors' contributions}

$A Z$ and $A A m$ drafted the article; $M G$ and $A M$ and $A A k$ gave contributions to interpretation of data for the study; RS gave substantial contributions to the acquisition, analysis, and interpretation of data for the study; ST and RS revised the article critically for important intellectual content; ST and SH finally approved the version of the article to be submitted. All authors read and approved the final manuscript.

\section{Availability of data and materials}

The datasets used and/or analysed during the current study are available from the corresponding author on reasonable request.

\section{Ethics approval and consent to participate}

The approval of the ethics committee had not been requested, in consideration of the retrospective study design.

\section{Consent for publication}

The manuscript does not contain any individual person's data in any form

\section{Competing interests}

None

\section{References}

1. Wu F, Zhao S, Yu B, et al. A new coronavirus associated with human respiratory disease in China. Nature. 2020;579(7798):265-9. 
2. Park C, Sugand K, Nathwani D, et al. Impact of the COVID-19 pandemic on orthopedic trauma workload in a London level 1 trauma center: the "golden month" The COVid Emergency Related Trauma and orthopaedics (COVERT) Collaborative. Acta orthopaedica. 2020;91(5):556-61.

3. Luceri F, Morelli I, Accetta R, et al. Italy and COVID-19: the changing patient flow in an orthopedic trauma center emergency department. BioMed Central; 2020;15:323.

4. Rodrigues-Pinto R, Sousa R, Oliveira A. Preparing to perform trauma and orthopaedic surgery on patients with COVID-19. The Journal of Bone and Joint Surgery American Volume. 2020;00:e1(1-5).

5. Egol KA, Konda SR, Bird ML, et al. Increased mortality and major complications in hip fracture care during the COVID-19 pandemic: a New York City perspective. Journal of orthopaedic trauma. 2020;00:1-8.

6. Porcel-Vazquez JA, Andres-Peiro JV, Garcia-Sanchez Y, et al. Usefulness of PCR screening in the initial triage of trauma patients during COVID-19 pandemic. Journal of orthopaedic trauma. 2020;00:e1-e5.

7. Long C, Xu H, Shen Q, et al. Diagnosis of the Coronavirus disease (COVID-19): rRT-PCR or CT? European journal of radiology. 2020;126:108961.

8. Farrell S, Schaeffer EK, Mulpuri K. Recommendations for the Care of Pediatric Orthopaedic Patients During the COVID Pandemic. The Journal of the American Academy of Orthopaedic Surgeons. 2020;00:1-10.

9. Hall AJ, Clement ND, Farrow L, et al. IMPACT-Scot report on COVID-19 and hip fractures: a multicentre study assessing mortality, predictors of early SARS-CoV-2 infection, and the effects of social lockdown on epidemiology. The Bone \& Joint Journal. 2020;102(9):1219-28.

10. Kayani B, Onochie E, Patil V, et al. The effects of COVID-19 on perioperative morbidity and mortality in patients with hip fractures: a multicentre cohort study. The Bone \& Joint Journal. 2020;102(9):1136-45.

11. Sadighi M, Mortazavi SMJ, Ebrahimpour A, et al. Fracture Surgery in known COVID-19 infected patients: what are the Challenges? Archives of Bone and Joint Surgery. 2020;8(3):378.

12. Mi $B$, Chen $L$, Xiong $Y$, et al. Characteristics and early prognosis of COVID-19 infection in fracture patients. The Journal of Bone and Joint Surgery American Volume. 2020;102(9):750.

13. Lei $\mathrm{S}$, Jiang F, Su W, et al. Clinical characteristics and outcomes of patients undergoing surgeries during the incubation period of COVID-19 infection. EClinicalMedicine. 2020;21:100331.

14. Huang C, Wang Y, Li X, et al. Clinical features of patients infected with 2019 novel coronavirus in Wuhan, China. The lancet. 2020;395(10223):497-506.

15. Lazizi M, Marusza CJ, Sexton SA, et al. Orthopaedic surgery in a time of COVID-19: Using a low prevalence COVID-19 trauma surgery model to guide a safe return to elective surgery. Bone \& Joint Open. 2020;1(6):229-35.

16. Jain VK, Lal H, Patralekh MK, et al. Fracture management during COVID-19 pandemic: a systematic review. Journal of Clinical Orthopaedics and Trauma. 2020;11:S431-S41. 
17. Zhu Y, Chen W, Xin X, et al. Epidemiologic characteristics of traumatic fractures in elderly patients during the outbreak of coronavirus disease 2019 in China. International Orthopaedics. 2020;44:156570.

18. Nuñez JH, Sallent A, Lakhani K, et al. Impact of the COVID-19 Pandemic on an Emergency Traumatology Service: Experience at a Tertiary Trauma Centre in Spain. Injury. 2020;51:1414-8.

19. Wang D, Hu B, Hu C, et al. Clinical characteristics of 138 hospitalized patients with 2019 novel coronavirus-infected pneumonia in Wuhan, China. Jama. 2020;323(11):1061-9.

20. Maniscalco P, Poggiali E, Quattrini F, et al. Proximal femur fractures in COVID-19 emergency: the experience of two Orthopedics and Traumatology Departments in the first eight weeks of the Italian epidemic. Acta Bio Medica: Atenei Parmensis. 2020;91(2):89.

21. Wu Z, McGoogan JM. Characteristics of and important lessons from the coronavirus disease 2019 (COVID-19) outbreak in China: summary of a report of 72314 cases from the Chinese Center for Disease Control and Prevention. Jama. 2020;323(13):1239-42.

22. Phua J, Weng L, Ling L, et al. Intensive care management of coronavirus disease 2019 (COVID-19): challenges and recommendations. The Lancet Respiratory Medicine. 2020;8(5):506-17.

\section{Tables}


Table 1

Baseline characteristics of all trauma patients with suspicious COVID-19 disease.

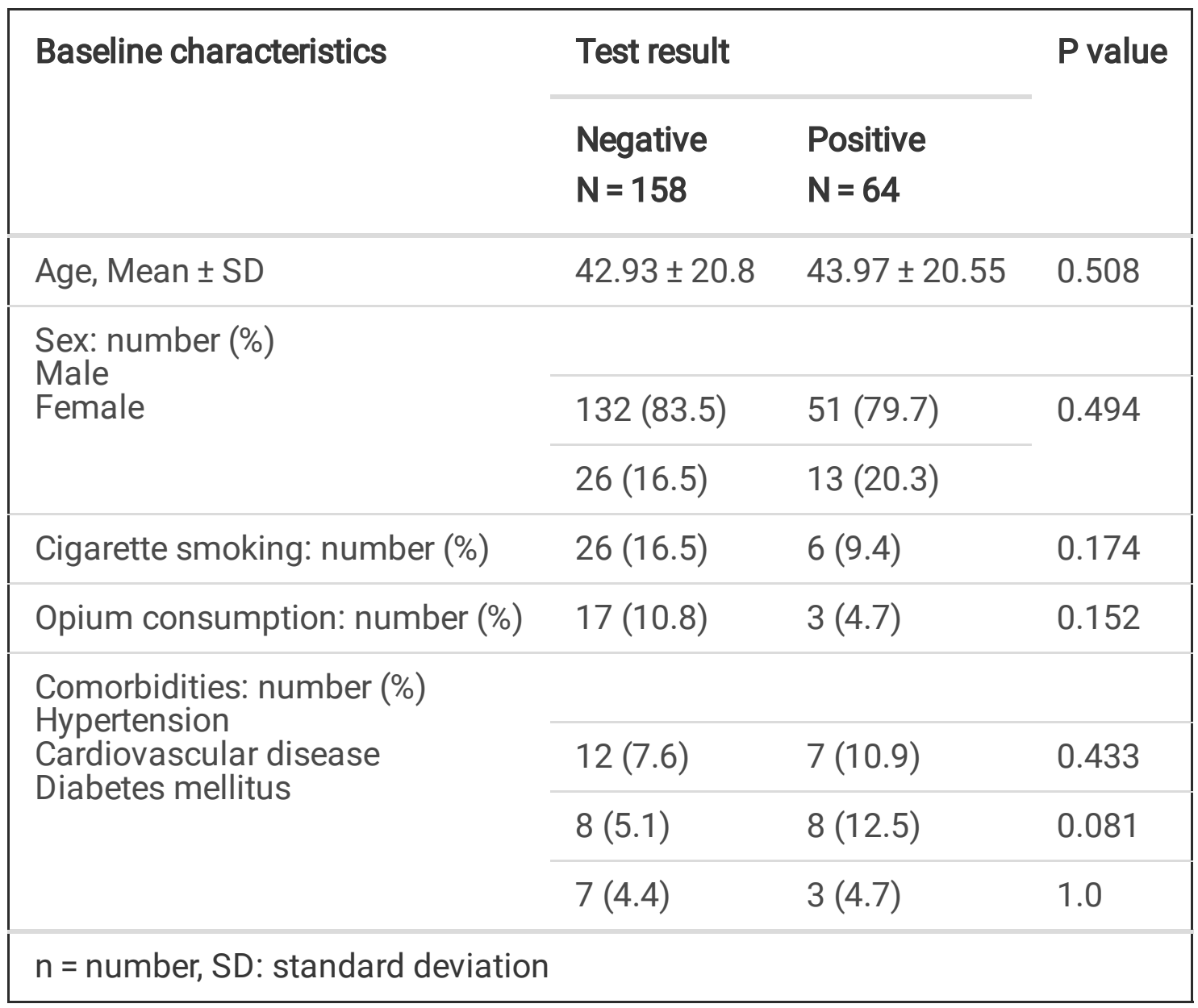


Table 2

Clinical data and outcome characteristics of all trauma patients with suspicious COVID-19 disease.

\begin{tabular}{|c|c|c|c|}
\hline \multirow{3}{*}{$\begin{array}{l}\text { Characteristics } \\
\text { Sign and symptom, n (\%) }\end{array}$} & \multicolumn{2}{|c|}{ Test result, $\mathrm{n}(\%)$} & \multirow[t]{3}{*}{$P$ value } \\
\hline & Negetive & Positive & \\
\hline & $N=158$ & $N=64$ & \\
\hline Fever & $60(38.0)$ & $24(37.5)$ & 0.947 \\
\hline Cough & $18(11.4)$ & $12(18.8)$ & 0.186 \\
\hline Weakness & $24(15.2)$ & $6(9.4)$ & 0.287 \\
\hline Anorexia & $9(5.7)$ & $1(1.6)$ & 0.288 \\
\hline Chest pain & $4(2.5)$ & $4(6.3)$ & 0.232 \\
\hline Body pain & $3(1.9)$ & $3(4.7)$ & 0.358 \\
\hline Headache & $3(1.9)$ & $1(1.6)$ & 1.0 \\
\hline Vertigo & $1(0.6)$ & $0(0)$ & 1.0 \\
\hline seizure & $0(0)$ & $1(0.4)$ & 0.288 \\
\hline History of exposure to a positive or suspicious case & $5(3.2)$ & $8(12.5)$ & 0.012 \\
\hline Destiny after admission & & & 0.899 \\
\hline ward & $24(15.2)$ & $9(14.1)$ & \\
\hline Isolated room & $54(34.2)$ & $24(37.5)$ & \\
\hline ICU & $80(50.6)$ & $31(48.4)$ & 0.286 \\
\hline Duration of hospitalization (day) & $8(1.2)$ & $6(1.3)$ & 0.347 \\
\hline outcome & & & 0.248 \\
\hline Discharged & $138(87.3)$ & $51(79.9)$ & \\
\hline Deceased & $16(10.1)$ & $10(15.6)$ & \\
\hline Remained & $4(2.5)$ & $3(4.7)$ & \\
\hline \multicolumn{4}{|l|}{ n:number } \\
\hline ICU: Intensive care unit & & & \\
\hline
\end{tabular}


Table 3

Analysis of the associations of baseline and demographic characteristics with ICU admission in patients presented with trauma and suspicious COVID-19 infection

\begin{tabular}{|c|c|c|c|c|}
\hline \multirow[t]{2}{*}{ Characteristics } & \multicolumn{4}{|c|}{ Location of admission } \\
\hline & ICU & Ward & p-value ${ }^{1}$ & p-value ${ }^{2}$ \\
\hline Age(mean) & 40.9 & 44.1 & 0.12 & 0.76 \\
\hline Mechanism of Injury: High/low & $76 / 24(76 / 24 \%)$ & $68 / 32(68 / 32 \%)$ & 0.21 & 0.24 \\
\hline $\begin{array}{l}\text { Sex } \\
\text { Male/female }\end{array}$ & $\begin{array}{l}91 / 20 \\
(82.9 / 17.1 \%)\end{array}$ & $\begin{array}{l}92 / 19 \\
(82 / 18 \%)\end{array}$ & 0.86 & 0.56 \\
\hline Cigarrette smoking & $22(19.8 \%)$ & $10(9 \%)$ & 0.02 & 0.097 \\
\hline Opium consumption & $14(12.6 \%)$ & $6(5.4 \%)$ & 0.06 & 0.93 \\
\hline COVID-19 & $31(27.9 \%)$ & $33(29.7 \%)$ & 0.77 & 0.95 \\
\hline $\begin{array}{l}\text { Comorbidities } \\
\text { HTN }\end{array}$ & $8(7.2 \%)$ & $11(9.9 \%)$ & 0.47 & 0.39 \\
\hline $\begin{array}{l}\text { CVD } \\
\text { DM }\end{array}$ & $\begin{array}{l}10(9 \%) \\
6(5.4 \%)\end{array}$ & $\begin{array}{l}6(5.4 \%) \\
4(3.6 \%)\end{array}$ & $\begin{array}{l}0.30 \\
0.52\end{array}$ & $\begin{array}{l}0.21 \\
0.59\end{array}$ \\
\hline Asthma & $2(1.8 \%)$ & $1(0.9 \%)$ & 0.56 & 0.52 \\
\hline \multicolumn{5}{|c|}{ ICU: Intensive care unit, HTN: hypertension, CVD: cardivascular disease DM: diabetes mellitus } \\
\hline \multicolumn{5}{|c|}{$\begin{array}{l}\text { 1. P-value related to the univariate analysis of the characteristics with mortality rate (mann-whitney } \\
\text { and chi square) }\end{array}$} \\
\hline \multicolumn{5}{|c|}{$\begin{array}{l}\text { 2. P-value related to the multivariate analysis of the characteristics with mortality rate (binary logistic } \\
\text { regression) }\end{array}$} \\
\hline
\end{tabular}


Table 4

Analysis of the associations of baseline and demographic characteristics with hospital

\begin{tabular}{|c|c|c|c|c|}
\hline \multirow[t]{2}{*}{ Characteristics } & \multicolumn{4}{|l|}{ Mortality } \\
\hline & expired & alive & p-value ${ }^{1}$ & p-value ${ }^{2}$ \\
\hline Age(mean) & 55.4 & 40.8 & 0.001 & 0.001 \\
\hline $\begin{array}{l}\text { Mechanism of Injury } \\
\text { High/low }\end{array}$ & $\begin{array}{l}16 / 6 \\
(72.7 / 27.3 \%)\end{array}$ & $\begin{array}{l}128 / 50 \\
(71.9 / 28.1 \%)\end{array}$ & 0.96 & 0.18 \\
\hline $\begin{array}{l}\text { Sex } \\
\text { Male/female }\end{array}$ & $\begin{array}{l}20 / 6 \\
(76.9 / 23.1 \%)\end{array}$ & $\begin{array}{l}163 / 33 \\
(83.2 / 16.8 \%)\end{array}$ & 0.43 & 0.40 \\
\hline Cigarette smoking & $6(23.1 \%)$ & $26(13.3 \%)$ & 0.18 & 0.27 \\
\hline Opium consumption & $5(19.2 \%)$ & $15(7.7 \%)$ & 0.05 & 0.09 \\
\hline Surgical procedure & $16(61.5 \%)$ & $100(51 \%)$ & 0.06 & 0.033 \\
\hline COVID-19 & $10(38.5 \%)$ & $54(27.6 \%)$ & 0.25 & 0.038 \\
\hline $\begin{array}{l}\text { Comorbidities } \\
\text { HTN }\end{array}$ & $4(15.4 \%)$ & $15(7.7 \%)$ & 0.19 & 0.76 \\
\hline $\begin{array}{l}\text { CVD } \\
\text { DM }\end{array}$ & $\begin{array}{l}5(19.2 \%) \\
2(7.7 \%)\end{array}$ & $\begin{array}{l}11(5.6 \%) \\
8(4.1 \%)\end{array}$ & $\begin{array}{l}0.012 \\
0.40\end{array}$ & $\begin{array}{l}0.20 \\
0.41\end{array}$ \\
\hline Asthma & $2(7.7 \%)$ & $1(0.5 \%)$ & 0.003 & 0.09 \\
\hline \multicolumn{5}{|c|}{ assocaited mortality rate in patients presented with trauma and suspicious COVID-19 } \\
\hline \multicolumn{5}{|c|}{ HTN: hypertension, CVD: cardivascular disease, DM: diabetes mellitus } \\
\hline \multicolumn{5}{|c|}{$\begin{array}{l}\text { 1. P-value related to the univariate analysis of the characteristics with mortality rate (mann-whitney } \\
\text { and chi square) }\end{array}$} \\
\hline \multicolumn{5}{|c|}{$\begin{array}{l}\text { 2. P-value related to the multivariate analysis of the characteristics with mortality rate (binary logistic } \\
\text { regression) }\end{array}$} \\
\hline
\end{tabular}


Table 5

The characteristics of the orthopedic trauma patients with confirmed COVID-19 disease.

\begin{tabular}{|ll|}
\hline Characterisitics & Value \\
\hline Mechanism, $\mathrm{n}(\%)$ & $24(72.7)$ \\
\hline Traffic accident & $9(27.3)$ \\
\hline Falling down & \\
\hline Orthopedic injury, $\mathrm{n}(\%)$ & \\
\hline Falling down & $3(0.09)$ \\
\hline Pertrochanteric fx & $3(0.09)$ \\
\hline Pelvic fx & $2(0.06)$ \\
\hline Spinal fx & $1(0.03)$ \\
\hline Femoral shaft fx & $1(0.03)$ \\
\hline Distal radius $\mathrm{fx}$ & $1(0.03)$ \\
\hline Tibia shaft $\mathrm{fx}$ & $1(0.03)$ \\
\hline Shoulder dx & $1(0.03)$ \\
\hline Elbow dx & $3(0.03)$ \\
\hline Accident & $3(0.09)$ \\
\hline Pelvic \&acetabulum $\mathrm{fx}$ & $3(0.21)$ \\
\hline Clavicle $\mathrm{fx}$ & $7(0.21)$ \\
\hline Femoral shaft fx & $4(0.12)$ \\
\hline Pertrochanteric $\mathrm{fx}$ & $3(0.09)$ \\
\hline Spinal fx & $3(0.06)$ \\
\hline Tibia shaft $\mathrm{fx}$ & $1(09)$ \\
\hline Humerus $\mathrm{fx}$ & \\
\hline Bimaleolar $\mathrm{fx}$ & \\
\hline Distal radius $\mathrm{fx}$ & \\
\hline
\end{tabular}

$\mathrm{n}=$ number, SD: standard deviation, Fx: fracture, ACL: anterior cruciate ligament Dx:dislocation 


\begin{tabular}{|lc|}
\hline Characterisitics & Value \\
\hline Both bone fx of forearm & $1(0.03)$ \\
\hline Bennet $f x$ & $1(0.03)$ \\
\hline Calcaneous fx & $1(0.03)$ \\
\hline Cuboid fx & $1(0.03)$ \\
\hline Patella fx & $1(0.03)$ \\
\hline ACL avulsion fx & $1(0.03)$ \\
\hline Scapula fx & $1(0.03)$ \\
\hline Surgical treatment of orthopedic injury, $\mathrm{n}(\%)$ & $23(0.70)$ \\
\hline Admission to surgery time interval, mean days (SD) & $8.2(6.8)$ \\
\hline Surgery duration, mean hours (SD) & $3.1(2.0)$ \\
\hline $\mathrm{n}=$ number, SD: standard deviation, Fx: fracture, ACL: anterior cruciate ligament \\
\hline Dx:dislocation & \\
\hline
\end{tabular}

\section{Figures}




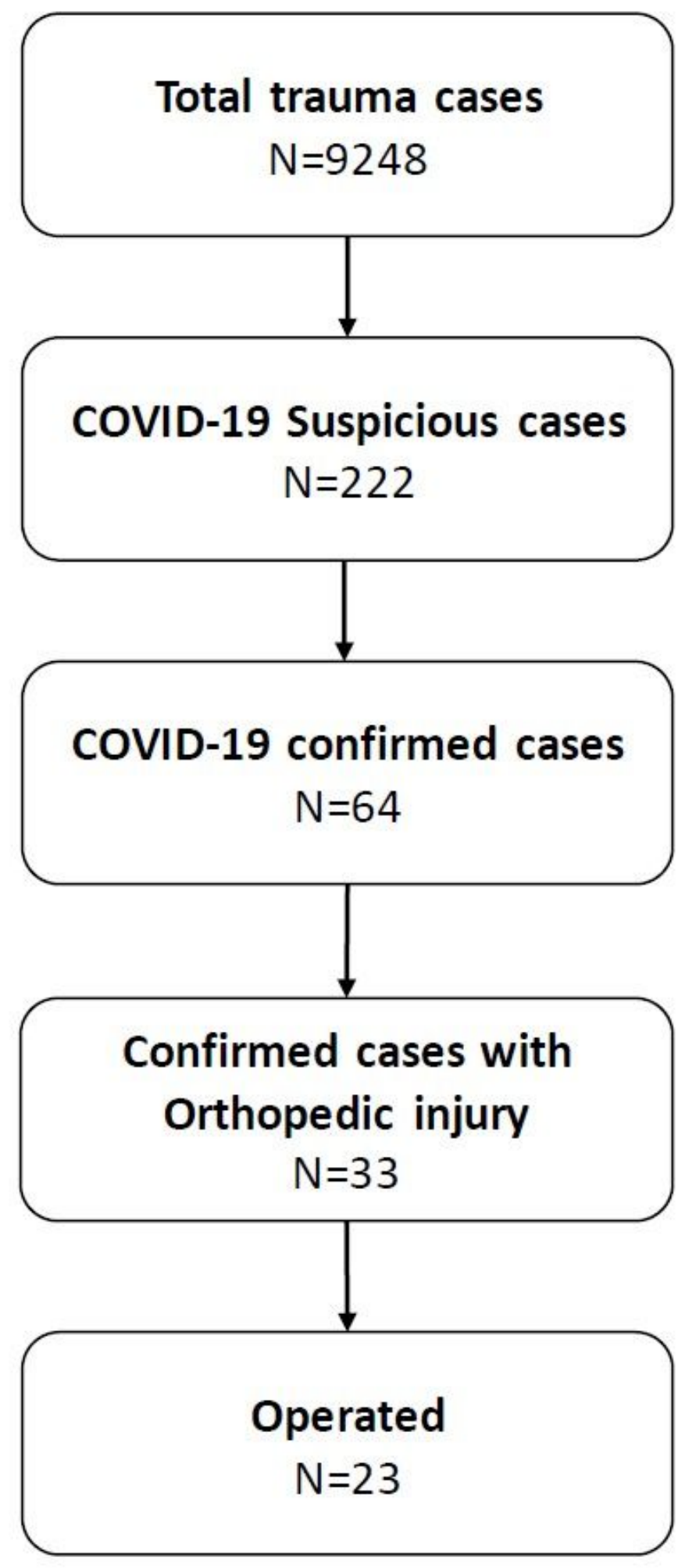

Figure 1

Diagram of orthopedic trauma patients during six months from COVID-19 pandemia in our referral trauma center. N: Number 